\title{
HMC algorithm for two-flavour lattice QCD: Schwarz-preconditioning with a one-dimensional domain decomposition
}

\author{
Martin Hasenbusch * \\ Dipartimento di Fisica dell'Università di Pisa and I.N.F.N., Largo Bruno Pontecorvo 3, \\ I-56127 Pisa, Italy \\ E-mail: Martin.Hasenbusch@df.unipi.it
}

\begin{abstract}
We study a variant of the Schwarz-preconditioned HMC algorithm. In contrast to the original proposal of Lüscher, we apply the domain decomposition in one lattice direction only. This is sufficient to reduce the condition number of the fermion matrix restricted to the domains compared with the full fermion matrix. For the same linear extension of the domain, less links reside on the boundaries of the domains. Therefore it becomes e.g. practical to iterate the decomposition. We perform numerical tests for two degenerate flavours of Wilson fermions. The standard Wilson gauge action at $\beta=5.6$ is used. The performance of our implementation is compared with other recent studies using various types of preconditioning.
\end{abstract}

The XXV International Symposium on Lattice Field Theory

July 30-4 August 2007

Regensburg, Germany

*Address after 31.10.2007: Institut für Theoretische Physik, Universität Leipzig, Postfach 100 900, D-04009 Leipzig, Germany 


\section{Introduction}

We consider a system with two degenerate flavours of quarks that is defined by the partition function

$$
Z=\int \mathrm{D}[U] \exp \left(-S_{G}[U]\right) \operatorname{det} M[U]^{2},
$$

where $S_{G}[U]=-\frac{\beta}{3} \sum_{x} \sum_{\mu>v} \operatorname{Re} \operatorname{Tr}\left(U_{x, \mu} U_{x+\hat{\mu}, v} U_{x+\hat{v}, \mu}^{\dagger} U_{x, v}^{\dagger}\right)$ is the standard Wilson plaquette action, $x=\left(x_{0}, x_{1}, x_{2}, x_{3}\right)$ with $x_{i}$ integer in the range $0 \leq x_{i}<L_{i}$ are sites on a hyper-cubical lattice, $\mu, v \in\{0,1,2,3\}$ are directions on the lattice and $\hat{\mu}$ is a unit vector in $\mu$-direction. The gaugefield $U_{x, \mu}$ is an element of the group $S U(3)$. In eq. (1.1), the fermion degrees of freedom have been integrated out, leading to the fermion determinant in the weight. The Wilson fermion matrix is given by

$$
M[U]_{x y}=1-\kappa \sum_{\mu}\left\{\left(1-\gamma_{\mu}\right) U_{\mu}(x) \delta_{x+\hat{\mu}, y}+\left(1+\gamma_{\mu}\right) U_{\mu}^{\dagger}(x-\hat{\mu}) \delta_{x-\hat{\mu}, y}\right\}
$$

where the $\gamma_{\mu}$ are the euclidian $\gamma$-matrices, and $\kappa$ is the so called hopping parameter, which is related with the bare mass of the fermions.

Recently there had been algorithmic progress $[1,2,3,4,5]$ in the simulation of lattice QCD at light quark masses. In two flavour simulations, following [6], the determinant of the fermion matrix $M$ is represented as $\operatorname{det} M M^{\dagger} \propto \int \mathrm{D} \phi^{\dagger} \int \mathrm{D} \phi \exp \left(-\left|M^{-1} \phi\right|^{2}\right)$, where $\phi$ is the pseudo-fermion field and $S_{p f}=\left|M^{-1} \phi\right|^{2}$ the pseudo-fermion action. The basic idea of $[1,2,3,4,5]$ is to chose alternative representations of the fermion determinant while keeping the Hybrid Monte Carlo (HMC) algorithm unchanged otherwise. To this end, the fermion matrix is factorized $M=\prod_{i} W_{i}$ such that the factors $W_{i}$ have a smaller condition number than the fermion matrix $M$ itself. A pseudo-fermion field is introduced for each of the factors

$$
\operatorname{det} M M^{\dagger}=\prod_{i=1}^{n} \operatorname{det} W_{i} W_{i}^{\dagger} \propto \int \mathrm{D} \phi_{1}^{\dagger} \int \mathrm{D} \phi_{1} \int \mathrm{D} \phi_{2}^{\dagger} \int \mathrm{D} \phi_{2} \ldots \int \mathrm{D} \phi_{n}^{\dagger} \int \mathrm{D} \phi_{n} \exp \left(-\sum_{i}\left|W_{i}^{-1} \phi_{i}\right|^{2}\right) .
$$

The effect of this splitting is two-fold: The noise of the stochastic representation of the fermion matrix is reduced compared with the standard pseudo-fermion action and furthermore, the splitting of the action allows to compute numerically expensive parts less frequently, as suggested in [7].

Here we discuss a variant of the Schwarz-preconditioned HMC put forward by Lüscher [4]. While in the other cases $[1,2,5]$ the factors $W_{i}$ can be written as a function of the fermion matrix, here a spatial decomposition is the basis for the factorization.

The lattice is decomposed into blocks of the size $l_{0} \times l_{1} \times l_{2} \times l_{3}$, with $l_{\mu}<L_{\mu}$. An approximation $W_{1}$ of $M$ is obtained by eliminating the hopping terms in $M$ that connect different blocks. Lüscher [4] made the important observation that $\operatorname{det}^{2}\left(W_{1}^{-1} M\right)$ can be estimated by using a pseudofermion field that resides on the boundaries of black blocks only (lets assume a red/black decomposition of the blocks.). Furthermore in eqs. (3.12,3.13) of [4] he shows how the force due to the pseudo-fermion action for $\operatorname{det}^{2}\left(W_{1}^{-1} M\right)$ can be computed efficiently. In the following we shall use these results without any modification; also the result of Appendix B of [4] is used in the following to reduce the dimension of the pseudo-fermion field by half.

Here we consider a block-decomposition in one dimension only, say the temporal direction. I.e. $l_{\mu}=L_{\mu}$ for $\mu=1,2,3$. The reasons to study this special case are the following: a) the 
implementation becomes much simpler; mainly because there are no sites in a corner of block. b) At least for the lattice spacings currently investigated, the fraction of links on the boundary between blocks is much less; therefore the number of active links, i.e. those links that take part in the molecular-dynamics evolution is larger. c) The simplification enables us to iterate the block decomposition.

Disadvantages of the one-dimensional decomposition are that it is less useful for a massive parallelization of the program and what might be more important, for the same $l_{0}$ the condition number of $W_{1}$ might be larger than for a decomposition in all four directions. However the experience with Schrödinger functional boundary conditions suggests that still there is a substantial reduction of the condition number of $W_{1}$ compared to $M$.

In our numerical experiments, we have iterated the decomposition twice. In the simulations discussed below, we have chosen $l_{0}^{(1)}=L_{0} / 2$ for the first step and $l_{0}^{(2)}=l_{0}^{(1)} / 2=L_{0} / 4$ for the second step of the decomposition. $W_{i}$ denotes the fermion matrix restricted to the blocks of size $l_{0}^{(i)}$. For $W_{2}$ we have used even-odd and mass-preconditioning [1]: $W_{3, e o}=W_{2, e o}+\rho$. I.e. the pseudofermion action consists of four parts: $S_{4}, S_{3}, S_{2}, S_{1}$ representing the squares of the determinant of $M W_{1}^{-1}, W_{1} W_{2}^{-1}, W_{2, e o} W_{3, e o}^{-1}$ and $W_{3, e o}$, respectively. Note the counter-intuitive connection between the labels of the $S$ and the $W . S_{0}$ is given by the gauge action.

\section{Integration with multiple time scales}

The basic steps of the integration scheme are given by

$$
T_{U}(\Delta \tau): U \rightarrow e^{i \Delta \tau P} U \quad \text { and } \quad T_{P, j}(\Delta \tau): P \rightarrow P-i \Delta \tau \delta_{U} S_{j}(U)
$$

where $\delta_{U}$ denotes a variation with respect to the gauge fields. From these basic steps we can build elementary leap-frog steps

$$
T_{L F, 0}\left(\Delta \tau_{0}\right)=T_{P, 0}\left(\frac{\Delta \tau_{0}}{2}\right) T_{U}\left(\Delta \tau_{0}\right) T_{P, 0}\left(\frac{\Delta \tau_{0}}{2}\right)
$$

or steps of an improved scheme (here we follow [7]):

$$
T_{S W, 0}\left(\Delta \tau_{0}\right)=T_{P, 0}\left(\lambda \Delta \tau_{0}\right) T_{U}\left(\frac{\Delta \tau_{0}}{2}\right) T_{P, 0}\left([1-2 \lambda] \Delta \tau_{0}\right) T_{U}\left(\frac{\Delta \tau_{0}}{2}\right) T_{P, 0}\left(\lambda \Delta \tau_{0}\right)
$$

with $\lambda=1 / 6$. Note that in an elementary step of this scheme, the variation of the action with respect to the gauge-fields has to be computed twice. This scheme is closely related with the second order minimum norm scheme ( $2 \mathrm{MN}$ ) studied in [8]. The only difference is the choice $\lambda \approx 1 / 5$ in [8]. $S_{0}$ is the part of the action with the largest forces. Elementary integration steps that include parts $S_{j}$ of the action that have smaller forces are now constructed recursively as

$$
T_{L F, j}\left(\Delta \tau_{j}\right)=T_{P, j}\left(\frac{\Delta \tau_{j}}{2}\right)\left[T_{X, j-1}\left(\Delta \tau_{j-1}\right)\right]^{n_{j-1}} T_{P, j}\left(\frac{\Delta \tau_{j}}{2}\right)
$$

in the leapfrog case and

$$
T_{S W, j}\left(\Delta \tau_{j}\right)=T_{P, j}\left(\lambda \Delta \tau_{j}\right)\left[T_{X, j-1}\left(\Delta \tau_{j-1}\right)\right]^{n_{j-1} / 2} T_{P, j}\left([1-2 \lambda] \Delta \tau_{j}\right)\left[T_{X, j-1}\left(\Delta \tau_{j-1}\right)\right]^{n_{j-1} / 2} T_{P, j}\left(\lambda \Delta \tau_{j}\right)
$$


in the improved case. The step sizes of the different levels are related as $\Delta \tau_{j}=n_{j-1} \Delta \tau_{j-1}$. In both cases $X$ can be either leap frog $(L F)$ or the improved scheme $(S W)$. This means that for different time scales, different integration schemes can be used. Here we have used the leapfrog scheme for the levels $j=2,3,4$ and the improved one for $j=0,1$. A full trajectory is given by $T_{L F, 4}\left(\Delta \tau_{4}\right)^{n_{4}}$.

In the case of the Schwarz-preconditioning, the force due to the pseudo-fermion action depends quite strongly on the position of the gauge link with respect to the boundaries of the blocks. I.e. here on $x_{0}$. Therefore, as discussed in [4], one might chose a step size that depends on the position, such that the step size times the force is roughly constant. As we shall see below, the force is the largest close to the boundaries of the blocks. Therefore, we have used the following schemes:

(A) In the case of $L_{0}=24$ we have used $s\left(x_{0}\right)=0.2,0.5,1,1,0.5$ and 2 for $x_{0}=0,1, \ldots, 5$ for the space-like links and $s\left(x_{0}\right)=0.2,0.5,1,0.5,0.2$ and 0 for $x_{0}=0,1, \ldots, 5$ for time-like links. This scheme is then repeated: $s\left(x_{0}+6 n\right)=s\left(x_{0}\right)$, where $n \in 1,2,3$.

(B) for $L_{0}=32$ is given by $s\left(x_{0}\right)=0,0.5,1,1,1,1,0.5$ and 0 , for $x_{0}=0,1, \ldots, 7$ for the space-like links and $s(0)=0,0.5,1,1,1,0.5,0$ and 0 for $x_{0}=0,1, \ldots, 7$, for time-like links. This scheme is then repeated: $s\left(x_{0}+8 n\right)=s\left(x_{0}\right)$, where $n \in 1,2,3$.

(C) for $L_{0}=32$ is given by $s(0)=0,0.25,0.5,1,1,1,1,0.25,0.25,1,1,1,1,0.5,0.25,0$, for $x_{0}=0,1, \ldots, 15$ for the spatial links and $s(0)=0,0,0.25,0.5,1,1,0.25,0,0.25,1,1,1,0.5,0.25$, 0,0 for $x_{0}=0,1, \ldots, 15$ for the time-like links. For $x_{0}>15: s\left(x_{0}\right)=s\left(x_{0}-16\right)$.

Note that the blocks of the first decomposition run from $x_{0}=0$ up to $L_{0} / 2-1$ and from $x_{0}=L_{0} / 2$ up to $L_{0}-1$. For the scheme (A) the average of $s$ over all links is 0.525 . For the schemes (B) and (C) it is about 0.59. The actual step size for a given link is $\Delta \tau$ quoted below times $s\left(x_{0}\right)$. In order to ensure ergodicity of the update, the configuration is shifted in time direction after each trajectory.

\section{Numerical results}

We have simulated the Wilson gauge action at $\beta=5.6$ with Wilson fermions using the values of the hopping parameter: $\kappa=0.1575,0.1580$ and 0.15825 . These parameters are chosen such that we can compare our results with $[4,5,9,10]$. Following the literature, these bare parameters correspond roughly to a pseudo-scalar mass of $690 \mathrm{MeV}, 490 \mathrm{MeV}$ and $370 \mathrm{MeV}$. Note that in the real world the pion mass is $m_{\pi} \approx 135 \mathrm{MeV}$. The lattice spacing is about $0.8 \mathrm{fm}$.

As solver we have used the geometric series for $S_{1}, S_{2}$ and $S_{3}$ and the BiCGstab solver with even-odd and Schwarz-preconditioning for $S_{4}$. The basic parameters of our runs are summarized in table 1 . The parameters of the algorithm have been chosen such that roughly the number of steps of the solver is the same for each part of the pseudo-fermion action. The typical length of our runs is 2000 trajectories after equilibration up to about 5000 trajectories for the runs with $L=12$. On 8 CPUs (Opteron $2.2 \mathrm{GHz}$ ) of a Cray XD1 computer one trajectory for the $32 \times 24^{3}$ lattice at $\kappa=0.15825$ took about 2.5 hours. Note that in our program the Dirac operator runs with less than one Gflops per processor and the sub-optimal choice of solver. Our CPU time can be compared with about 0.3 hours [4] (from fig. 7) on 8 nodes with two $2.4 \mathrm{GHz}$ Xeon CPUs each. Note that in this case the trajectory length is only $\tau=0.5$ and also the number of active links is about half of ours. 
Table 1: Basic parameters of our runs. $P_{a c c}$ is the acceptance rate at the end of the trajectory. $S$ denotes the scheme used for the $x_{0}$ dependence of the step size. $\rho$ is the parameter of the mass preconditioning.

\begin{tabular}{|c|c|c|c|c|l|l|l|l|l|l|}
\hline$L_{0}$ & $L=L_{1}=L_{2}=L_{3}$ & $\mathrm{~S}$ & $\kappa$ & $\rho$ & $n_{4}$ & $n_{3}$ & $n_{2}$ & $n_{1}$ & $n_{0}$ & \multicolumn{1}{|c|}{$P_{a c c}$} \\
\hline 24 & 12 & $\mathrm{~A}$ & 0.1575 & 0.15 & 6 & 1 & 2 & 2 & 4 & $0.892(2)$ \\
24 & 12 & $\mathrm{~A}$ & 0.1580 & 0.15 & 6 & 1 & 2 & 2 & 4 & $0.916(2)$ \\
32 & 16 & $\mathrm{~B}$ & 0.1575 & 0.20 & 4 & 1 & 3 & 2 & 4 & $0.704(5)$ \\
32 & 16 & $\mathrm{~B}$ & 0.1580 & 0.15 & 5 & 1 & 3 & 2 & 4 & $0.826(4)$ \\
32 & 16 & $\mathrm{~B}$ & 0.15825 & 0.15 & 5 & 1 & 3 & 2 & 4 & $0.826(4)$ \\
32 & 24 & $\mathrm{~B}$ & 0.15825 & 0.15 & 7 & 1 & 3 & 2 & 4 & $0.83(2)$ \\
32 & 24 & $\mathrm{C}$ & 0.15825 & 0.15 & 5 & 3 & 2 & 2 & 4 & $0.875(4)$ \\
\hline
\end{tabular}

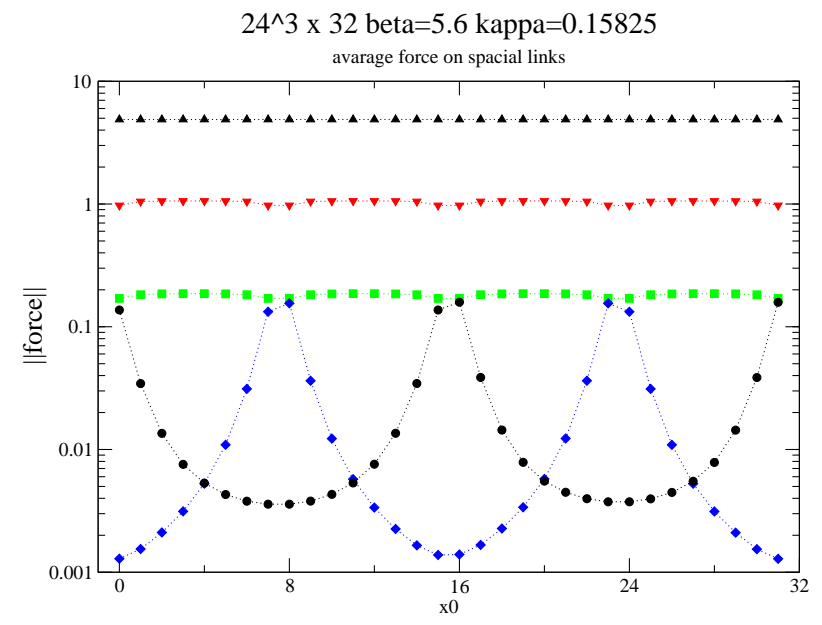

Figure 1: We give the average force on spatial links as a function of $x_{0}$. For a discussion see the text

In fig. 1 we give the average forces on the spatial links as a function of $x_{0}$. The largest force is obtained for the gauge action. The forces due to $S_{3}$ and $S_{4}$ display a strong dependence on $x_{0}$. They are largest at the boundaries between the blocks. In the case of $S_{4}$, they assume their minimum in the middle of the block. In the case of $S_{3}$ the minimum is located at the boundaries of the blocks of the first decomposition. Note that the minimum of the force due to $S_{3}$ is much smaller than that of the force due to $S_{4}$.

The step sizes needed to obtain a sufficient acceptance rate can be compared with results from the literature. Here we give only a small selection: Using standard HMC, the authors of [10] need the step size $\Delta \tau=0.006$ for $\kappa=0.1580$ on a $32 \times 16^{3}$ lattice to get $P_{a c c}=0.66$. Note that in this case the pseudo-fermion action is computed with the fermion matrix itself and not with the evenodd preconditioned one. Our most difficult case, the $32 \times 24^{3}$ lattice at $\kappa=0.15825$ we compare with [4] who needs $\Delta \tau=0.05$ to reach $P_{a c c}=0.86$ and [9], using mass preconditioning, where $\Delta \tau=0.1$ is needed to get $P_{a c c}=0.8$. In [5] $\Delta \tau=0.25$ is used in combination with a fourth order minimal norm integrator. 
$16^{\wedge} 3 \times 32$ beta $=5.6 \mathrm{kappa}=0.1575$

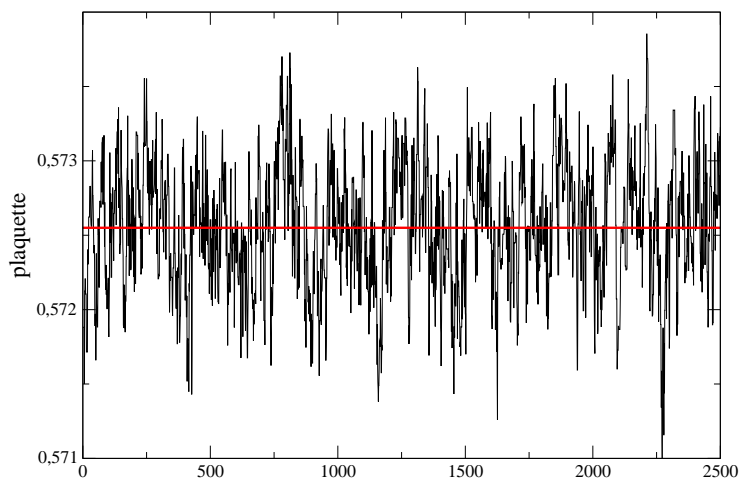

$24^{\wedge} 3 \times 32$ beta $=5.6 \mathrm{kappa}=0.15825$

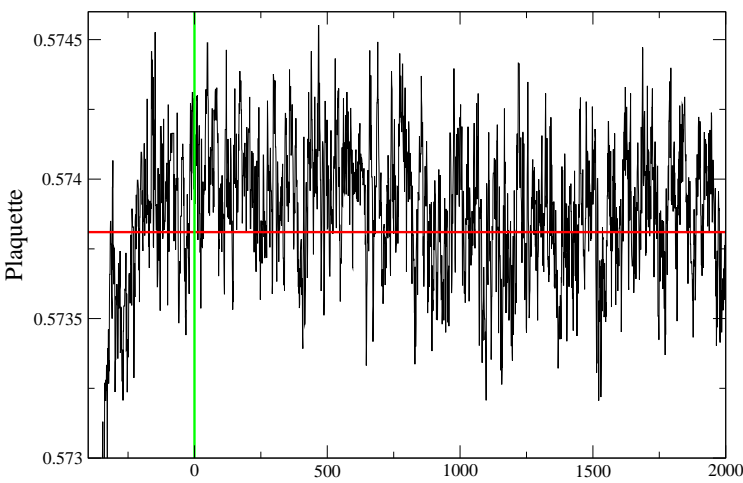

$16^{\wedge} 3 \times 32$ beta $=5.6$ kappa $=0.1575$

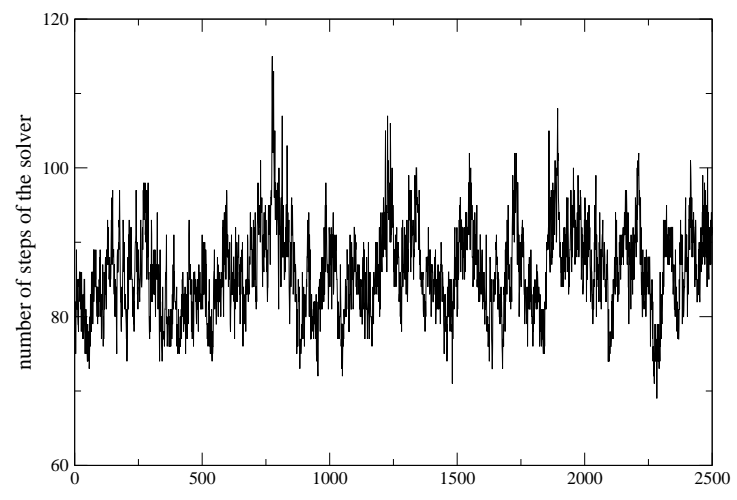

$24^{\wedge} 3 \times 32$ beta $=5.6$ kappa $=0.15825$

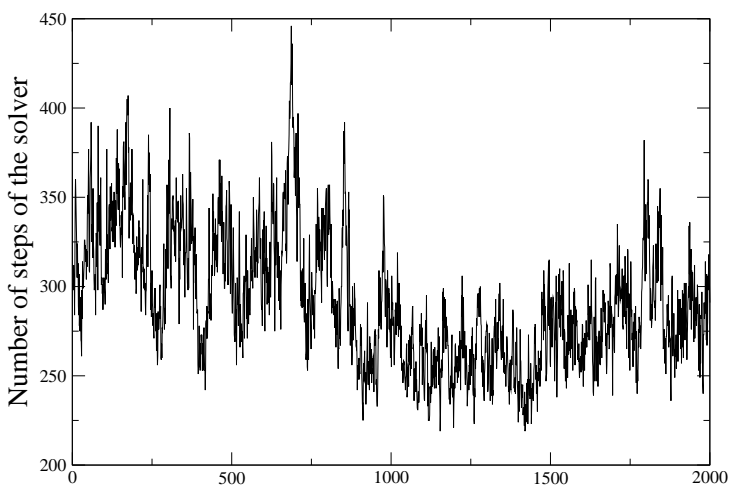

Figure 2: History of the plaquette average and the number of steps of the solver. The red line gives the average of the plaquette obtained in [10] in the case of $L=16$ and $\kappa=0.1575$ and $[4,12]$ in the case of $L=24$ and $\kappa=0.15825$.

In order to judge the performance of an algorithm autocorrelation times for the quantities of interest have to be determined. This is however a notoriously hard problem in HMC simulations of QCD with dynamical fermions.

In fig. 2 we give the evolution of the plaquette value and the number of steps taken by the solver for the simulation of a $32 \times 16^{3}$ lattice at $\kappa=0.1575$. The run started from a configuration equilibrated by a different version of HMC algorithm. The plots give no indication for autocorrelation times that are comparable with the length of the run itself. We get $\tau_{P}=8(2)$ and $\tau_{s o l v}=16(5)$ as integrated autocorrelation times of the plaquette and the number of steps of the solver. The time unit is given by a trajectory. These numbers can be compared with $\tau_{P}=7(4)$ and $\tau_{\text {solv }}=33(4)$ for a standard HMC simulation [10] and $\tau_{P}=68(25)$ and $\tau_{\text {solv }}=168(42)$ for a Schwarz preconditioned HMC simulation [4]. Note that in [4] the trajectory length is $\tau=0.5$ and only about $37 \%$ of the links are active. This might trivially explain a factor of about 4 compared with our simulation. One also should note that the authors of [11] find that even larger trajectory lengths such as $\tau=2$ are advisable to obtain optimal performance.

In the case of $L=24$ and $\kappa=0.15825$ we do not quote values for autocorrelation times. The time histories of the average plaquette and the number of solver steps suggests that there are correlations that are comparable with the length of our run or even larger. Note that the authors of 
$[4,5,9,12]$ do not see such problems and quote rather small values of the autocorrelation times. Taking into account the length of the trajectory and the fraction of active links, our run is of similar length as that of [12]. One should take into account the possibility that $[4,5,9,12]$ do not see these large autocorrelations since their runs are too short.

\section{Conclusions and outlook}

Using preconditioned pseudo-fermion actions $[1,2,3,4,5]$ the problem that the step size needed to obtain a reasonable acceptance rate decreases with decreasing fermion mass seems to be overcome. The performance of the different proposals seems to be quite similar. Still the dependence of autocorrelation times related to small eigenvalues of the fermion matrix on the choice of the pseudo-fermion action is not well understood. To this end, it might be useful to monitor e.g. the topological charge. Likely also much longer runs then those presented here and in $[5,9,12]$ are needed to this end. A disadvantage of the Schwarz-preconditioning is that it is quite hard to implement fermion actions that are more complicated than clover-improved Wilson fermions. Since in the case of Schwarz-preconditioning the pseudo-fermions reside on boundaries only, it is possible that the performance of the HMC scales differently (hopefully better) with the lattice spacing than for the other types of preconditioning.

\section{References}

[1] M. Hasenbusch, Phys.Lett. B 519 (2001) 177 [arXiv:hep-lat/0107019].

[2] M. Peardon, Nucl.Phys.B (Proc.Suppl.) 106\&107 (2002) 3 [arXiv:hep-lat/0201003].

[3] M. Hasenbusch and K. Jansen, Nucl.Phys.B 659 (2003) 299 [arXiv:hep-lat/0211042].

[4] M. Lüscher, JHEP 0305 (2003) 052 [arXiv:hep-lat/0304007]; Comput.Phys.Commun. 165 (2005) 199 [arXiv:hep-lat/0409106].

[5] M.A. Clark and A.D. Kennedy, Phys.Rev.Lett. 98 (2007) 051601 [arXiv:hep-lat/0608015].

[6] D. Weingarten and D. Petcher, Phys. Lett. B 99 (1981) 333.

[7] J.C. Sexton and D.H. Weingarten, Nucl.Phys.B 380 (1992) 665.

[8] T. Takaishi and P. de Forcrand, Phys.Rev.E 73 (2006) 036706 [arXiv:hep-lat/0505020].

[9] C. Urbach, K. Jansen, A. Shindler and U. Wenger, Comput.Phys.Commun. 174 (2006) 87 [arXiv:hep-lat/0506011].

[10] B. Orth, T. Lippert and K. Schilling, Phys.Rev.D 72 (2005) 014503 [arXiv:hep-lat/0503016] and refs therein.

[11] H.B. Meyer, H. Simma, R. Sommer, M. Della Morte, O. Witzel and U. Wolff, Comput.Phys.Commun. 176 (2007) 91 [arXiv:hep-lat/0606004].

[12] L. Del Debbio, L. Giusti, M. Lüscher, R. Petronzio and N. Tantalo, JHEP 0602 (2006) 011 [arXiv:hep-lat/0512021]; JHEP 0702 (2007) 056 [arXiv:hep-lat/0610059]; M. Lüscher, private communication. 